\title{
MEASURES SATISFYING A REFINED DOUBLING CONDITION AND ABSOLUTE CONTINUITY
}

\author{
ALF JONSSON
}

(Communicated by Andrew Bruckner)

\begin{abstract}
It is shown that for certain subsets $F \subset \mathbb{R}^{n}$, two measures with support $F$ satisfying a refined doubling condition are necessarily mutually absolutely continuous. This is contrary to the situation with measures satisfying the usual doubling condition, in which case no such result is available.
\end{abstract}

\section{INTRODUCTION}

Let $F$ be a closed nonempty subset of $\mathbb{R}^{n}, 0<s \leq n$, and denote by $B(x, r)$ the open ball with center $x$ and radius $r$. A positive Borel measure $\mu$ with support $F$ satisfies the condition $D_{s}$ if $\mu$ is finite on finite sets and there is a constant $c>0$ such that

$$
\mu(B(x, k r)) \leq c k^{s} \mu(B(x, r)), \quad x \in F, k \geq 1, k r \leq 1 .
$$

In this paper we show that if $F$ is a $d$-set with $d=s$ and $\mu_{1}$ and $\mu_{2}$ are measures with support $F$ satisfying $D_{s}$, then $\mu_{1}$ and $\mu_{2}$ are mutually absolutely continuous. For the definition of a $d$-set we refer to Section 2 , but mention here as examples that the closure of a Lipschitz domain in $\mathbb{R}^{n}$ is a $d$-set with $d=n$, and the usual Cantor ternary set is a $d$-set with $d=\ln 2 / \ln 3$. In Section 4 it is pointed out how the result for $d$-sets can be used to obtain generalizations to more general sets $F$.

In [4] it is shown that if $F$ is a compact subset of $\mathbb{R}^{n}$, then there always exists a positive Borel measure $\mu$ with support $F$ satisfying the condition $D_{n}$ and thus in particular the doubling condition

$$
\mu(B(x, 2 r)) \leq c \mu(B(x, r)), \quad x \in F, 0<r \leq 1 / 2 .
$$

The question whether two measures with support $F$ which satisfy the doubling condition are mutually absolutely continuous is discussed in [4], and it is noted by referring to an example by A. Beurling and L. Ahlfors that this is not the case even when $F$ is an interval. The results of the present paper show that replacing the doubling condition by the refined doubling condition $D_{s}$, we get mutual absolute continuity for classes of closed sets $F$.

Received by the editors December 1, 1993.

1991 Mathematics Subject Classification. Primary 28A12.

This work was supported by the Swedish Natural Science Research Council.

(c)1995 American Mathematical Society 
For the background to the study of measures satisfying the refined doubling condition $D_{s}$ and for applications of such measures we refer to [2] and [4].

\section{Classes of measures}

The notation $D_{s}$ for measures satisfying (1) is the same as in [4], except that in that paper a global condition is used; the condition $k r \leq 1$ is not imposed. It is clear that if $\mu$ satisfies the condition $D_{s}$, then it satisfies the doubling condition (2). The converse holds in the sense that if $\mu$ satisfies the doubling condition, then it satisfies $D_{s}$ for some $s$. To see this take an integer $i$ so that $2^{i-1}<k \leq 2^{i}$, let $c$ be as in (2), and put $s_{0}=\ln c / \ln 2$. Then $\mu(B(x, k r)) \leq c^{i} \mu\left(B\left(x, k 2^{-i} r\right)\right) \leq c^{i} \mu(B(x, r))$ where $c^{i}=2^{i s_{0}}=2^{s_{0}} 2^{(i-1) s_{0}} \leq$ $2^{s_{0}} k^{s_{0}}$, so (1) is fulfilled with $s=s_{0}$. Note also that if $\mu$ satisfies (1), then $\mu(B(x, 1))=\mu(B(x,(1 / r) r)) \leq c r^{-s} \mu(B(x, r))$, so the condition $D_{s}$ implies

$$
\mu(B(x, r)) \geq c r^{s} \mu(B(x, 1)), \quad 0<r \leq 1 .
$$

A measure $\mu$ which satisfies

$$
c_{1} r^{d} \leq \mu(B(x, r)) \leq c_{2} r^{d}, \quad x \in F, 0<r \leq 1,
$$

for some positive constants $c_{1}$ and $c_{2}$ is called a $d$-measure on $F$. If $F$ is the support of a $d$-measure, then $F$ is a $d$-set. Any two $d$-measures on a $d$-set $F$ are equivalent. If $F$ is a $d$-set, then the restriction to $F$ of the $d$-dimensional Hausdorff measure is a $d$-measure on $F$. We denote this measure by $m$ and use it as a canonical $d$-measure on $F$. For the theory of $d$-measures, including proofs of the above results, see [3].

We conclude this section by giving an example of a measure satisfying the condition $D_{n}$ taking $n=1$ and $F$ as the closed interval $[0,1]$. Of course, the Lebesgue measure provides such an example, but we want to exhibit a less trivial one. Let the function $f$ be given by $f(x)=1 / \sqrt{x}, 0<x<1$, and $f(x)=0$ elsewhere, and put $d \mu=f d x$. Then $\mu$ satisfies $D_{1}$; we sketch a proof of this. Let $x_{0} \in[0,1]$ and $R>0$. If $R \leq x_{0}$, then $\mu\left(\left(x_{0}-R, x_{0}\right)\right) \leq$ $\mu\left(B\left(x_{0}, R\right)\right) \leq 2 \mu\left(\left(x_{0}-R, x_{0}\right)\right)$ and $\mu\left(\left(x_{0}-R, x_{0}\right)\right)=2\left(\sqrt{x}_{0}-\sqrt{x_{0}-R}\right)=$ $2 R /\left(\sqrt{x}_{0}+\sqrt{x_{0}-R}\right)$, so

$$
R / \sqrt{x}_{0} \leq \mu\left(B\left(x_{0}, R\right)\right) \leq 4 R / \sqrt{x}_{0} .
$$

The latter inequality in this formula holds for $R>x_{0}$, too, since then $\mu\left(B\left(x_{0}, R\right)\right) \leq \mu\left(\left(0, x_{0}+R\right)\right) \leq 2 \sqrt{x_{0}+R}=2\left(x_{0}+R\right) / \sqrt{x_{0}+R} \leq 4 R / \sqrt{x}_{0}$. Thus, if $r \leq x_{0}$ and $k r \leq 1$ we have $\mu\left(B\left(x_{0}, k r\right)\right) \leq 4 k r / \sqrt{x}_{0} \leq 4 k \mu\left(B\left(x_{0}, r\right)\right)$. If instead $r>x_{0}$, then $\mu\left(B\left(x_{0}, k r\right)\right) \leq \mu((0,(k+1) r)) \leq 2 \sqrt{(k+1) r}=$ $\sqrt{k+1} \mu((0, r)) \leq 2 k \mu\left(B\left(x_{0}, r\right)\right)$, and thus we have shown that $\mu$ satisfies $D_{1}$.

\section{Mutual absolute continuity}

It is well known (see, e.g., [4]) that there exists a measure $\mu$ defined on $[0,2 \pi]$ which is singular with respect to the one-dimensional Lebesgue measure and satisfies the doubling condition, so two measures with support on a compact set $F \subset \mathbb{R}^{n}$ satisfying the doubling condition need not be mutually absolutely continuous. Since, as we saw, a measure satisfying the doubling condition also satisfies the condition $D_{s}$ if $s$ is big enough, this also shows, if we 
consider the interval $[0,2 \pi]$ as a subset of some $\mathbb{R}^{n}$ with $n$ big enough, that two measures satisfying the condition $D_{n}$ on a closed subset $F \subset \mathbb{R}^{n}$ need not be mutually absolutely continuous. Therefore, in order to get absolute continuity for measures satisfying the refined doubling condition, one has to impose restrictions on $F$. We work in this section with an $n$-set $F$, or more generally, corresponding to the condition $D_{d}$, a $d$-set $F$, and give some generalizations in the next section.

Assume that $F$ is a $d$-set with canonical $d$-measure $m$, and that $\mu$ is a measure with support $F$ satisfying $D_{d}$. From (3) it follows that $\mu(B(x, r)) \geq$ $c r^{d}, 0<r \leq 1$, as long as $x$ belongs to some compact set $K \subset F$, for if $\inf _{x \in K} \mu(B(\bar{x}, 1))=0$, then there exists a point $x_{0} \in K$ and a sequence $x_{k} \rightarrow$ $x_{0}, k \rightarrow \infty$, such that $\mu\left(B\left(x_{0}, 1 / 2\right)\right) \leq \mu\left(B\left(x_{k}, 1\right)\right) \rightarrow 0, k \rightarrow \infty$, which contradicts the assumption that $\mu$ has support $F$. Combining this with (4) we get that

$$
m(B(x, r)) \leq c \mu(B(x, r)), \quad x \in K, 0<r \leq 1 .
$$

But this means that $m$ is absolutely continuous with respect to $\mu, m<<\mu$ for short, as can be seen by the following argument. Let $\mu(E)=0$, take a compact subset $K$ of $E$, and, given $\epsilon>0$, an open set $O \supset K$ with $\mu(O)<\epsilon$, and cover $K$ with finitely many balls $B\left(x_{i}, r\right) \subset O, i=1,2, \ldots, i_{0}$. Take a subset $S$ of these balls such that the balls in $S$ are disjoint and the balls $3 B, B \in S$, cover $K$. (This can be achieved by choosing the first ball to be in $S$ as a ball from the covering with largest radius, then omitting all balls from the covering which intersect the first chosen ball, taking as a second ball in $S$ a ball with largest radius among the remaining balls, and so on.) Then we have

$$
m(K) \leq \sum_{B \in S} m(3 B) \leq c \sum_{B \in S} m(B) \leq c \sum_{B \in S} \mu(B) \leq c \mu(O) \leq c \epsilon,
$$

so we must have $m(K)=0$ and consequently $m(E)=0$.

Thus we have shown that if $F$ is a $d$-set with $d$-measure $m$ and $\mu$ is a measure with support $F$ satisfying the condition $D_{d}$, then $m<<\mu$. The following theorem shows that we also have that $\mu<<m$.

Theorem 1. Let $0<d \leq n$, and let $F \subset \mathbb{R}^{n}$ be a $d$-set with canonical $d$ measure $m$. Assume that $\mu$ is a measure with support $F$ satisfying the condition $D_{d}$. Then $\mu$ is absolutely continuous with respect to $m$.

Before proving the theorem we note that in view of the discussion above the following corollary holds.

Corollary 1. Let $0<d \leq n$, and let $F \subset \mathbb{R}^{n}$ be a $d$-set. Assume that $\mu_{1}$ and $\mu_{2}$ are measures with support $F$ satisfying the condition $D_{d}$. Then $\mu_{1}$ and $\mu_{2}$ are mutually absolutely continuous.

Proof of Theorem 1. Put

$$
D \mu(x)=\lim _{r \rightarrow 0} \frac{\mu(B(x, r))}{m(B(x, r))}
$$

at every point in $F$ where the limit exists, and call the limit $f(x)$ at those points. Then $D \mu$ exists $m$-a.e. and the function $f$ is locally integrable with respect to $m$; see, e.g., [1] (actually $f$ equals the Radon-Nikodym derivative 
of the part of $\mu$ which is absolutely continuous with respect to $m)$. Put $A=$ $\{x \in F: D \mu(x)$ exists $\}$.

Let $x_{0} \in A$. Then, given $\epsilon>0$ there is a $\delta>0$ such that

$$
\left|\frac{\mu\left(B\left(x_{0}, r\right)\right)}{m\left(B\left(x_{0}, r\right)\right)}-f\left(x_{0}\right)\right|<\epsilon, \quad r \leq \delta,
$$

and hence

$$
\mu\left(B\left(x_{0}, r\right)\right)<m\left(B\left(x_{0}, r\right)\right)\left(f\left(x_{0}\right)+\epsilon\right), \quad r \leq \delta .
$$

For $\delta<r \leq 1$ we get, using that $\mu$ satisfies $D_{d}$, the formula (5), and that $m$ is a $d$-measure, $\mu\left(B\left(x_{0}, r\right)\right)=\mu\left(B\left(x_{0},(r / \delta) \delta\right)\right) \leq c(r / \delta)^{d} \mu\left(B\left(x_{0}, \delta\right)\right) \leq$ $c(r / \delta)^{d} m\left(B\left(x_{0}, \delta\right)\right)\left(f\left(x_{0}\right)+\epsilon\right) \leq c(r / \delta)^{d} \delta^{d}\left(f\left(x_{0}\right)+\epsilon\right)$, and so since $m$ is a $d$-measure,

$$
\mu\left(B\left(x_{0}, r\right)\right) \leq c m\left(B\left(x_{0}, r\right)\right)\left(f\left(x_{0}\right)+\epsilon\right), \quad \delta<r \leq 1 .
$$

Combining this inequality with (5) we see that it holds for $0<r \leq 1$, and letting $\epsilon \rightarrow 0$ we get

$$
\mu\left(B\left(x_{0}, r\right)\right) \leq c m\left(B\left(x_{0}, r\right)\right) f\left(x_{0}\right), \quad 0<r \leq 1, x_{0} \in A .
$$

Now let $E \subset F$ be a set with $m(E)=0$. We shall show that $\mu(E)=0$, which proves the theorem.

We assume first that $F$ is compact. Put $A_{N}=\{x \in A \cap F: f(x) \leq N\}$, and fix an $\epsilon>0$. Choose $N=N(f, \epsilon)>0$ so big that $A_{N}$ is nonempty and $\int_{F \backslash A_{N}} f d m<\epsilon$; then $N m\left(F \backslash A_{N}\right) \leq \int_{F \backslash A_{N}} f d m<\epsilon$, so

$$
m\left(F \backslash A_{N}\right)<\epsilon / N \text {. }
$$

Next take $K$ compact satisfying $K \subset E$ and $\mu(K) \geq \frac{1}{2} \mu(E)$, and choose an open set $O$ so that $K \subset O$ and

$$
m(O)<\epsilon / N \text {. }
$$

Cover $K$ with balls contained in $O$ with centers in $K$, and choose a finite subcovering consisting of distinct balls $B_{\nu}=B\left(x_{\nu}, r_{\nu}\right), \nu=1,2, \ldots, \nu_{0}$. Let

$$
r_{\nu}^{\prime}=\max \left(r_{\nu}, \sup \left\{r: B\left(x_{\nu}, r\right) \cap A_{N}=\varnothing\right\}\right)
$$

and put $C_{\nu}=B\left(x_{\nu}, r_{\nu}^{\prime}\right)$ so that $B_{\nu} \subset C_{\nu}$. Choose $x_{\nu}^{\prime} \in A_{N}$ in $2 C_{\nu}$ and let $C_{\nu}^{\prime}=B\left(x_{\nu}^{\prime}, 5 r_{\nu}^{\prime}\right)$; then $3 C_{\nu} \subset C_{\nu}^{\prime}$.

Choose now a ball with largest radius among the balls $C_{\nu}, \nu=1,2, \ldots, \nu_{0}$, call it $S_{1}$, and denote the corresponding $C_{\nu}^{\prime}$ by $S_{1}^{\prime}$. Put $\mathscr{M}_{1}=\left\{C_{\nu}: C_{\nu} \cap\right.$ $\left.S_{1} \neq \varnothing\right\}$. Then our choice of $S_{1}$ guarantees that $C_{\nu} \subset S_{1}^{\prime}$ if $C_{\nu} \in \mathscr{M}_{1}$. Choose next $S_{2}$ as a ball with largest radius among the balls $C_{\nu} \notin \mathscr{M}_{1}$, and put $\mathscr{M}_{2}=\left\{C_{\nu} \notin \mathscr{M}_{1}: C_{\nu} \cap S_{2} \neq \varnothing\right\}$, and so on. Thus, in the $i$ th step, $i>1$, we choose $S_{i}$ as the ball with largest radius among the balls $C_{\nu} \notin \cup_{j=1}^{i-1} \mathscr{M}_{j}$ and put $\mathscr{M}_{i}=\left\{C_{\nu} \notin \cup_{j=1}^{i-1} \mathscr{M}_{j}: C_{\nu} \cap S_{i} \neq \varnothing\right\}$. The procedure stops at some $i \leq \nu_{0}$, say at $i_{0}$. Then the balls $S_{i}$ are disjoint and the balls $S_{i}^{\prime}, i=1,2, \ldots, i_{0}$, cover $K$. Let $I_{1}=\left\{i, 1 \leq i \leq i_{0}: S_{i}\right.$ equals some $\left.B_{\nu}\right\}$ and $I_{2}=\left\{i, 1 \leq i \leq i_{0}: i \notin I_{1}\right\}$. Then

$$
\sum_{i \in I_{1}} m\left(S_{i}\right)<m(O)<\epsilon / N
$$


since $S_{i} \subset O$ if $i \in I_{1}$ and

$$
\sum_{i \in I_{2}} m\left(S_{i}\right)<m\left(F \backslash A_{N}\right)<\epsilon / N
$$

since by construction the balls $S_{i}, i \in I_{2}$, are all in the complement of $A_{N}$.

Now, since $x_{\nu}^{\prime} \in A_{N}$, and because we may assume that things have been arranged in such a way that the radii $r_{\nu}^{\prime}$ are all small, we get from (6) that

$$
\mu\left(S_{i}^{\prime}\right) \leq c N m\left(S_{i}^{\prime}\right) .
$$

Consequently, since $S_{i}^{\prime} \subset 7 S_{i}$ and $m$ satisfies the doubling condition,

$$
\sum_{i} \mu\left(S_{i}^{\prime}\right) \leq c N \sum_{i} m\left(S_{i}^{\prime}\right) \leq c N \sum_{i} m\left(S_{i}\right) \leq c N(2 \epsilon / N)=c \epsilon,
$$

so $\mu(K) \leq c \epsilon$, where $c$ does not depend on $\epsilon$. Thus we must have $\mu E=0$ since $\mu(K) \geq \frac{1}{2} \mu(E)$.

If $F$ is not compact, one has to do some modifications. Let $X=F \cap\{x$ : $|x| \leq R\}$ and $X_{1}=F \cap\{x:|x| \leq R+1\}$, where $R>0$ is chosen so big that $X$ has positive $m$-measure. It is enough to prove that $\mu(X \cap E)=0$. This time let $A_{N}=\left\{x \in A \cap X_{1}: f(x) \leq N\right\}$ and choose $N$ so that $m\left(X_{1} \backslash A_{N}\right)<\epsilon / N$. Take a compact set $K \subset X \cap E$ and proceed as before. Then the balls $S_{i}, i \in I_{2}$, are all in $\{x:|x| \leq R+1\} \backslash A_{N}$ and the proof works as before.

\section{AN EXAMPLE}

Theorem 1 can be generalized to more general sets $F$ than $d$-sets. Let $F$ be a closed subset of $\mathbb{R}^{n}$, let $\mu$ be a measure with support $F$ satisfying the condition $D_{d}$, and let $m$ be the restriction to $F$ of the $d$-dimensional Hausdorff measure. Suppose that closed subsets $F_{k}, k=1,2, \ldots$, of $F$ can be chosen that satisfy the following conditions:

(1) $F_{k} \subset F_{k+1}, k=1,2, \ldots$,

(2) the sets $F_{k}$ are $d$-sets,

(3) the measures $\mu_{k}=\mu \mid F_{k}$ satisfy the condition $D_{d}$, and

(4) the set $G:=F \backslash F_{0}$ where $F_{0}=\cup_{k=1}^{\infty} F_{k}$ has measure zero with respect to the measures $\mu$ and $m$.

Let $E$ be a set with $m(E)=0$. Then $\mu(E)=\mu(E \cap G)+\mu\left(E \cap F_{0}\right)=\mu\left(E \cap F_{0}\right)=$ $\lim _{k \rightarrow \infty} \mu\left(E \cap F_{k}\right)=0$, where the last equality follows from Theorem 1 since $m\left(E \cap F_{k}\right)=0$. Thus $\mu<<m$, and in the same way one shows $m<<\mu$. We exemplify how to use these observations in an explicit example.

Example. Let $\gamma>1$, and let $F \subset \mathbb{R}^{2}$ be given by $F=\left\{\left(x_{1}, x_{2}\right): 0 \leq x_{1} \leq\right.$ $\left.1,0 \leq x_{2} \leq x_{1}^{\gamma}\right\}$. Then $F$ is not a $d$-set for some $d$. Take $F_{k}$ as $\{x \in F$ : $\left.x_{1} \geq 1 /(k+2)\right\}$, let $m$ be the Lebesgue measure, and let $\mu$ be a measure with support $F$ satisfying $D_{2}$. We shall see that the conditions (1)-(4) above are satisfied with $d=2$. Thus $\mu<<m$, and if $\mu_{1}$ and $\mu_{2}$ are two measures with support $F$, then they are mutually absolutely continuous.

Clearly (1) holds and (2) follows since $F_{k}$ is the closure of a Lipschitz domain. To check (3), take $x \in F_{k}$. Let $\mu_{k}=\mu \mid F_{k}$ and $k r \leq 1$. Then $\mu_{k}(B(x, k r)) \leq \mu(B(x, k r)) \leq c k^{2} \mu(B(x, r / 4))$. If $B(x, r / 4)$ does not intersect $F \backslash F_{k}$ we are done, since then

$$
\mu(B(x, r / 4))=\mu_{k}(B(x, r / 4)) \leq \mu_{k}(B(x, r)) .
$$


If $B(x, r / 4)$ intersects $F \backslash F_{k}$ we use that the ball $B\left(x_{0}, r / 8\right), x_{0}=x+$ $(r / 8,0)$, does not intersect $F \backslash F_{k}$. Then $\mu(B(x, r / 4)) \leq \mu\left(B\left(x_{0}, r / 2\right)\right) \leq$ $c \mu\left(B\left(x_{0}, r / 8\right)\right)=c \mu_{k}\left(B\left(x_{0}, r / 8\right)\right) \leq c \mu_{k}(B(x, r))$, which is what we want.

Finally (4) is seen in the following way. Since $\mu\left(\left\{x: 0<x_{1}<1 / k\right\}\right) \rightarrow$ $0, k \rightarrow \infty$, and $\mu\{(0,0)\} \leq \mu\left(B\left(p_{k}, 1 / k\right)\right) \leq c \mu\left(B\left(p_{k}, 1 /(2 k)\right)\right) \leq c \mu(\{x: 0<$ $\left.\left.x_{1}<1 / k\right\}\right)$ where $p_{k}=(1 /(2 k), 0)$, we must have $\mu\{(0,0)\}=0$.

\section{REFERENCES}

1. A.S. Besicovitch, $A$ general form of the covering principle and relative differentiation of additive functions, Proc. Cambridge Philos. Soc. 41 (1945), 103-110; II, Proc. Cambridge Philos. Soc. 42 (1946), 1-10.

2. A. Jonsson, Besov spaces on closed subsets of $\mathbb{R}^{n}$, Trans. Amer. Math. Soc. 341 (1994), 355-370.

3. A. Jonsson and H. Wallin, Function spaces on subsets of $\mathbb{R}^{n}$, Mathematical Reports, vol. 2, Part 1, Harwood Acad. Publ., Chur, Switzerland, 1984.

4. A.L. Volberg and S.V. Konyagin, On measures with the doubling condition, Izv. Akad. Nauk CCCP Ser. Mat. 51 (1987); English transl., Math USSR-Izv. 30 (1988), 629-637.

Department of Mathematics, University of UmeÃ, 90187 UmeẢ, SWeden

E-mail address: alf jon@math.dept.umu. se 